\title{
Working Memory Plasticity in Old Age: Practice Gain, Transfer, and Maintenance
}

\author{
Shu-Chen Li \\ Max Planck Institute for Human Development \\ Oliver Huxhold \\ Max Planck Institute for Human Development \\ and German Center of Gerontology \\ Jacqui Smith \\ Max Planck Institute for Human Development \\ and University of Michigan, Ann Arbor
}

\author{
Florian Schmiedek \\ Max Planck Institute for Human Development \\ and Humboldt University \\ Christina Röcke \\ Max Planck Institute for Human Development \\ and University of Zurich \\ Ulman Lindenberger \\ Max Planck Institute for Human Development
}

\begin{abstract}
Adult age differences in cognitive plasticity have been studied less often in working memory than in episodic memory. The authors investigated the effects of extensive working memory practice on performance improvement, transfer, and short-term maintenance of practice gains and transfer effects. Adults age 20-30 years and 70-80 years practiced a spatial working memory task with 2 levels of processing demands across 45 days for about $15 \mathrm{~min}$ per day. In both age groups and relative to age-matched, no-contact control groups, we found (a) substantial performance gains on the practiced task, (b) near transfer to a more demanding spatial $n$-back task and to numerical $n$-back tasks, and (c) 3-month maintenance of practice gains and near transfer effects, with decrements relative to postpractice performance among older but not younger adults. No evidence was found for far transfer to complex span tasks. The authors discuss neuronal mechanisms underlying adult age differences and similarities in patterns of plasticity and conclude that the potential of deliberate working memory practice as a tool for improving cognition in old age merits further exploration.
\end{abstract}

Keywords: working memory, plasticity, cognitive training, transfer, aging

Plasticity, or the potential of brain and behavior to reorganize in response to environmental opportunities and demands, characterizes organisms of all ages, albeit to varying degrees (Baltes, Lindenberger, \& Staudinger, 2006; Bialystok \& Craik, 2006; Lerner, 1984; Li, 2003; Lindenberger, Li, \& Bäckman, 2006). In

Shu-Chen Li and Ulman Lindenberger, Center for Lifespan Psychology, Max Planck Institute for Human Development, Berlin, Germany; Florian Schmiedek, Center for Lifespan Psychology, Max Planck Institute for Human Development, and Department of Psychology, Humboldt University, Berlin, Germany; Oliver Huxhold, Center for Lifespan Psychology, Max Planck Institute for Human Development, and German Center of Gerontology, Berlin; Christina Röcke, Center for Lifespan Psychology, Max Planck Institute for Human Development, and Department of Psychology, University of Zurich, Zurich, Switzerland; Jacqui Smith, Center for Lifespan Psychology, Max Planck Institute for Human Development, and University of Michigan, Ann Arbor.

Oliver Huxhold is now at the German Center of Gerontology, Berlin. Christina Röcke is now at the Department of Psychology, University of Zurich, Zurich, Switzerland. Jacqui Smith is now at the Department of Psychology and the Institute of Social Research, University of Michigan.

Correspondence concerning this article should be addressed to ShuChen Li, Center for Lifespan Psychology, Max Planck Institute for Human Development, Lentzeallee 94 D14195, Berlin, Germany. E-mail: shuchen@mpib-berlin.mpg.de humans, cognitive interventions are a powerful tool for exploring age-graded changes in plasticity (Hertzog, Kramer, Lindenberger, \& Wilson, in press; Lindenberger \& Baltes, 1995). Intervention research in cognitive aging has focused on episodic memory and fluid intelligence (for reviews, see Baltes et al., 2006; Kramer \& Willis, 2002; Verhaeghen, Marcoen, \& Goossens, 1992). Only very recently has research on cognitive plasticity in old age been extended to working memory and executive functions (Brehmer, Westerberg, Söderman, Fischer, \& Bäckman, 2008; Dahlin, Stigsdotter-Neely, Larsson, Bäckman, \& Nyberg, 2008; Karbach \& Kray, 2008).

In cognitive interventions, performance improvements on trained or practiced tasks should be charted and additional design features should be included to delineate whether more profound alterations in the organization of behavior have occurred. In this context, transfer and maintenance assessments are particularly relevant. To examine the scope and magnitude of plastic changes in behavior, researchers should administer transfer tasks that vary in the degree of processing overlap with the trained or practiced task before and after the intervention. In addition, maintenance of intervention effects on the trained or practiced tasks and the transfer tasks should be assessed. Without information on transfer and maintenance, it is difficult for one to discern whether intervention effects are restricted to the formation of a new skill, with little consequences for behavior unrelated to this 
skill, or whether cognitive mechanisms and capacities of general applicability have been enhanced. For instance, in the domain of working memory, interventions may result in the acquisition of a narrow set of skills for carrying out one particular type of working memory tasks without enhancement of working memory capacity in general.

In the domain of episodic memory, available evidence shows that cognitively healthy older adults (a) are able to acquire skilled episodic memory performance through instruction and training of mnemonic strategies (Verhaeghen et al., 1992); (b) show less improvement and lower performance asymptotes than do younger adults (Baltes \& Kliegl, 1992; Kliegl, Smith, \& Baltes, 1989; Singer, Lindenberger, \& Baltes, 2003; Verhaeghen et al., 1992) and children 9-12 years of age (Brehmer, Li, Müller, Oertzen, \& Lindenberger, 2007; Shing, Werkle-Bergner, Li, \& Lindenberger, 2008); (c) are able to maintain skilled memory performance for up to several months and, in some cases, even years (Brehmer et al., 2008; Stigsdotter Neely \& Bäckman, 1993); (d) generally either show no transfer or show transfer limited to tasks amenable to the trained memory skill (for reviews, see Rebok, Carlson, \& Langbaum, 2007; Verhaeghen et al., 1992). Similar findings have been observed for fluid intelligence, in which transfer, if it occurs at all, is limited to tasks that share surface features with the practiced or trained tasks. This fact allows for direct application of newly acquired or reactivated task-specific skills or strategies (Ball et al., 2002; for reviews, see Baltes et al., 2006; Baltes \& Lindenberger, 1988; Kramer \& Willis, 2002).

Adult age differences in the plasticity of working memory have rarely been studied. Given the substantial age-related decline in working memory (e.g., Borella, Carretti, \& de Beni, 2008; Craik, 1983; Paxton, Barch, \& Rachine, 2008) and the central role of working memory in cognition (e.g., Baddeley, 2007; Barrett, Tugade, \& Engle, 2004; Oberauer, 2005), the lack of research on working memory plasticity in old age is somewhat surprising. If working memory in old age is plastic to a substantial degree, positive transfer to fluid intelligence, which is closely related to working memory (Kane et al., 2004; Kyllonen \& Christal, 1990; Salthouse \& Pink, 2008; Süss, Oberauer, Wittmann, Wilhelm, \& Schulze, 2002) and to everyday competence, would seem within reach.

With a few recent exceptions (Brehmer et al., 2008; Dahlin et al., 2008; Karbach \& Kray, 2008), research on the plasticity of working memory and executive functions has focused on younger adults (e.g., Jaeggi, Buschkuehl, Jonides, \& Perrig, 2008; Olesen, Westerberg, \& Klingberg, 2004) and children (Klingberg et al., 2005). In younger adults, extensive daily practice over weeks in a spatial working memory task (Olesen et al., 2004) or a combined spatial and verbal working memory task (Jaeggi et al., 2008) resulted in positive transfer to measures of fluid intelligence, such as the Raven's Progressive Matrices Test (Jaeggi et al., 2008). Olesen et al. (2004) found that younger adults' spatial working memory capacity increased after 5 weeks of practice in three visuospatial working memory tasks. Reaction times decreased over the course of the intervention, and this suggested experienceinduced increments in processing speed.

In the study by Dahlin et al. (2008), younger and older adults practiced a working memory task that presumably put high demands on the information-updating aspect of working memory. Both younger and older adults improved on the practiced task. A transfer effect to another working memory task that also required continuous updating (i.e., a three-back task) was found in younger adults but not in older adults. In younger adults, brain activations in the striatum during performance of the trained task and the transfer task were substantially increased after the intervention. In older adults, intervention-related activation changes in the striatum were restricted to the practiced task and were absent from the transfer task. Hence, Dahlin et al. (2008) concluded that the observed age differences in transfer are connected to age-related changes in the striatum, which has been proposed to be specifically involved in updating operations (O'Reilly \& Frank, 2006).

This study investigated adult age differences in working memory plasticity demonstrated after extensive, deliberate practice in a spatial working memory task with two levels of processing demands. In particular, we examined age differences (a) in performance gains on the practiced task, (b) near and far transfer, and (c) short-term maintenance of both practice gains and transfer effects. The target working memory task was practiced 15 min per day for 45 days and resulted in an unusually high and distributed dosage of potentially plasticity-inducing experience in a well-controlled experimental setting. Given the current lack of relevant evidence, our study was guided by general expectations rather than specific hypotheses. We expected (a) performance improvements on the practiced task in both age groups, reflective of the preserved ability of older adults to acquire a new skill; (b) preserved but presumably less transfer in older adults than in younger adults; and (c) preserved but presumably lower levels of short-term maintenance on the practiced task in older relative to younger adults. Furthermore, we were especially interested in knowing whether transfer effects would be maintained over time, especially in older adults.

\section{Method}

We used an experimental-plus-control group design with a 3-month follow-up to investigate working memory plasticity and its maintenance in younger and older adults. The experimental group underwent 45 daily practice sessions. This design allowed us to ascertain the extent of practice-related effects beyond test-retest gains as well as to tap into short-term maintenance of these effects. To investigate transfer effects, we assessed the participants' performances after training in several tasks. They ranged from specific working memory tasks that shared some processing operations with the practiced task (near transfer tasks) over complex memory span tasks (far transfer tasks) to simple decision speed tasks for assessing nonmemory-specific transfer.

\section{Sample}

Recruitment of participants for the experimental and control groups was conducted through (a) newspaper and radio advertisements, (b) draws from the participant pool of the Center for Lifespan Psychology at the Max Planck Institute for Human Development, and (c) referrals of other participants already recruited for the study. Participants in both the experimental and control groups agreed, at the outset, that they would be willing to participate in a study with 45 daily sessions. After this agreement, control group participants were asked whether they would be willing to participate in a smaller study comprising only two sessions of assessment. Thus, although our recruitment scheme 
was not based on random assignment, it effectively implemented a high degree of similarity between experimental and control groups regarding demographic characteristics and motivation to participate in the study.

The experimental group comprised 19 younger adults (20-30 years, $M=25.3$ years, $S D=2.8)$ and 21 older adults $(70-80$ years, $M=74.5$ years, $S D=2.8$ ). Of these individuals, 11 younger and 15 older adults later also participated in the 3-month maintenance session, which took place on average about 12 weeks after posttest. The no-contact control group comprised 27 younger adults (21-30 years, $M=26.4$ years, $S D=2.4)$ and 20 older adults $(70-80$ years, $M=73.3$ years, $S D=2.8)$. There were no significant differences between the experimental and control groups with respect to mean age, $F(1,44)=2.17$ for younger and $F(1,39)=2.14$ for older adults, and the variance of age, $F(1$, $44)=1.99$ for younger and $F(1,39)=0.14$ for older adults. Furthermore, at baseline the experimental and control groups performed comparably in the digit symbol substitution test, which is a common indicator of processing speed. No significant differences in the mean or variance of performance were found between the experimental and control groups in younger adults $\left(M_{\exp }=\right.$ $60.0, S D_{\text {exp }}=11.5$ vs. $\left.M_{\text {contr }}=61.6, S D_{\text {contr }}=9.1\right), F(1,44)=$ 0.30 ; Levene's test, $F(1,44)=0.88$. Similarly, there were no differences between control and experimental groups in older adults $\left(M_{\exp }=37.4, S D_{\exp }=8.7\right.$ vs. $M_{\text {contr }}=40.1, S D_{\text {contr }}=$ 9.2), $F(1,38)=.91$; Levene's test, $F(1,38)=0.00$. Participants were paid 10 euros per hour of assessment. For a successful completion of the daily assessment period, which was defined as providing data for at least 40 (out of 45) daily sessions, the participants received an additional bonus of 200 euros. Thirtythree participants in the experimental group completed all 45 daily sessions, and the other 7 completed between 42 and 44 sessions.

\section{Procedure}

Participants in the experimental group completed a pretest assessment, which was distributed over 3 consecutive days before the practice sessions started. A posttest assessment was completed in one testing session after the daily practice phase. The pretest assessment comprised a large battery of self-report measures of personality and well-being, health, psychometric intelligence, and physical and sensorimotor functioning. In addition to measures of spatial working memory, the daily assessments included self-report measures of wellbeing, stress, and health as well as measures of postural control, vigilance, and perceptual speed (see Huxhold, 2007; Röcke, 2006, for details). These other measures are not included in the current analysis, as they do not directly pertain to the themes addressed in this article. In the posttest session, we assessed participants with the target task to investigate practice gains and with a range of near and far transfer tasks to examine the extents of transfer effects. Participants in the experimental group also took part in a 3-month follow-up maintenance session. Data collection for the control group was conducted after the experimental group had completed the 45 daily practice sessions.

The time between pretest and posttest for younger participants on average was 83 days $(S D=3.4)$ for the experimental group and 77 days $(S D=2.1)$ for the control group. For the older participants, it was 78 days $(S D=3.8)$ for the experimental group and 77 days $(S D=0.9)$ for the control group. Average time between posttest and maintenance sessions was 86 days $(S D=5.0)$ for the younger group and 86 days $(S D=6.3$ ) for the older group.

\section{Practice and Transfer Tasks}

Practice tasks. In each daily session, participants first worked on four blocks of trials of a spatial two-back task in a regular condition and then worked on four blocks of a more demanding condition that required mental spatial shifting and updating of memory information. In the regular condition, a sequence of black circles appeared in the eight outer squares of a three-by-three grid (the middle square was not used). The participant's task was to respond "yes" if the position of the circle in the current trial matched the one seen two trials before and to respond "no" if there was no match. In the shifting condition, circles appeared in the same manner as in the regular condition, but
A

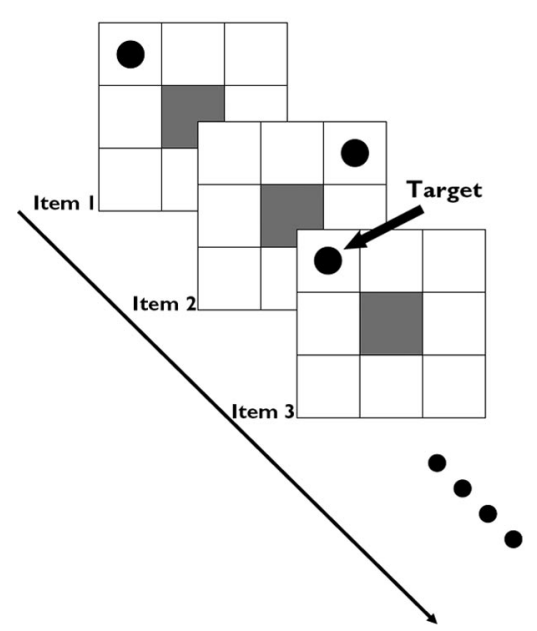

B

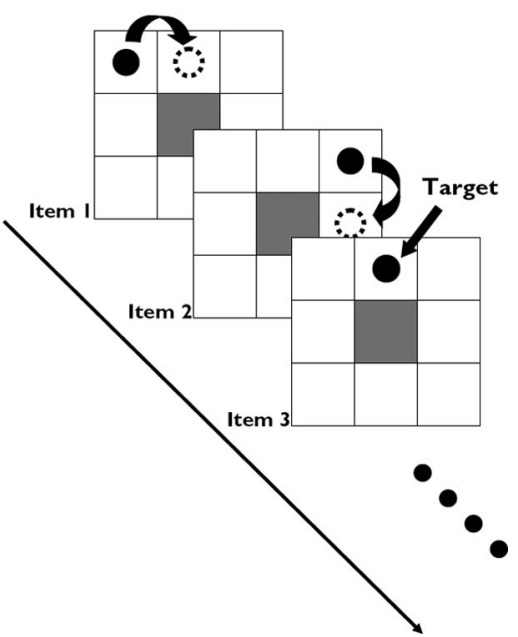

Figure 1. Schematic diagram of the spatial $n$-back task in the regular (A) and the more demanding mental shifting (B) conditions. 
the participants had to mentally shift the presented position one step clockwise and memorize the shifted positions (see Figure 1 for a schematic depiction of the two task conditions). The participant's task in this case was to respond "yes" if the position of the circle on the current trial matched the shifted position of the circle seen two trials before. In both conditions, participants were instructed to respond as fast and as accurately as possible.

In both conditions, each block consisted of 22 trials (i.e., 88 trials across four blocks per condition per day in total), which were randomly drawn with the constraint that two circles in consecutive steps could not be in the same position. The random sequences of the trials differed across the 45 daily sessions but were identical for all participants within a given session. The presentation time for each circle was $500 \mathrm{~ms}$, and interstimulus intervals (ISI) equaled 2,500 ms. Participants had to respond with their right index finger on a key marked green for targets and with their left index finger on a key marked red for nontargets. Each day, participants received feedback about their mean reaction times and overall accuracy on the working memory task.

Overview of transfer tasks. Transfer tasks were selected such that they allowed us to ascertain the generality of practice effects. We included tasks that assessed near and far transfer. For near transfer to a more demanding task with same memory content, we included a spatial three-back task that shared processing requirements with the practiced task but at a higher level of working memory load. For near transfer to tasks with different memory content, we included two numerical $n$-back tasks that shared processing requirements with the practiced task but in the verbal instead of the spatial modality. For far transfer, we included two complex memory span tasks. Last, we included two simple decision speed tasks, one with spatial and one with numerical materials, to assess potential speed transfer that was not memory specific. All transfer tasks were assessed during pre- and posttest sessions.

Near transfer: $n$-back working memory transfer tasks. Each of the transfer tasks was assessed with three blocks, each of which consisted of 22 trials (i.e., with a total of 66 trials). Two types of $n$-back transfer tasks were selected to assess near transfer. One was the spatial working memory transfer task, generated from the practiced tasks by increasing memory load from two back to three back. The others were the two-back and three-back numerical working memory transfer tasks generated by changing the memory content (modality) of the to-be-remembered material from spatial to numerical materials. The spatial tasks used the same three-bythree grid, whereas the numerical tasks involved single-digit numbers from 0 to 9 . Presentation times of the stimuli were $500 \mathrm{~ms}$, and ISIs were fixed to $2,500 \mathrm{~ms}$ for all tasks. We used these tasks to further specify observed transfer effects to the three-back spatial and to the two- and three-back numerical tasks.

Far transfer: complex span tasks. We included two complex span tasks to assess far transfer: operation span (Turner \& Engle, 1989) and rotation span (Shah \& Miyake, 1996). These tasks do not share common processing requirements with the $n$-back tasks in any obvious way. In the operation span task, an equation verification task was combined with a short-term memory task for one-syllable nouns. The equations were similar to "Is (4/2) $-1=$ 1 ?" and were displayed simultaneously with the nouns that appeared to the right of the equation. Participants had to press the green key for correct equations and the red key for incorrect equations. After each sequence of equations (sequence length defined the memory load, which varied from 3 to 6), participants had to recall the nouns in the correct order to the experimenter, who wrote them down. Presentation times for the equations and nouns were the time participants took to respond to the equation (with a maximum of 2,000 ms) plus $1,500 \mathrm{~ms}$ after the response. ISI was $500 \mathrm{~ms}$. After two practice trials, there were 12 trials to be completed, three per load level. Percentages of correctly recalled words at correct serial positions were calculated for each trial and then averaged across trials.

The rotation span task combined a letter-rotation task with the short-term memory requirement of remembering the letter orientations. A sequence of regular or mirror-reversed letters was displayed on the screen. Letters were rotated by 45, 90, 135, 180, 225 270 , or 315 degrees. Participants were asked to press the green key when letters were displayed regularly and the red key when the letters were mirror reversed. In addition, participants had to memorize the orientation of the letter. At the end of each sequence, participants had to recall the orientations of the letters in the order of their presentation and to indicate them by filling in numbers on a paper layout with the seven possible directions denoted by dots arranged in a circle. Presentation times for the letters were 3,000 $\mathrm{ms}$, and ISIs equaled $500 \mathrm{~ms}$. Sequence length (load level) varied from two to five. After two practice trials, there were 12 trials to be completed, three per load level. Percentages of correctly recalled orientations at correct serial positions were calculated for each trial and averaged across trials.

Non-memory-specific speed transfer: simple decision speed tasks. To keep the stimuli and response demands as similar as possible, we made the design of the spatial and numerical twochoice reaction-time tasks (e.g., numbers of trials, stimulus presentations, and response requirements) identical to that of the two-back working memory task, except that working memory load was at a minimum (i.e., zero back; henceforth labeled as zero-back tasks). In the spatial version, participants had to decide whether the stimulus that appeared in any of the eight outer cells of the three-by-three grid was a black or a white circle. In the numerical version, participants had to decide whether the number that appeared in the center cell of the three-by-three grid was a 1,2, or 3 versus any other number between 4 and 9 .

\section{Results}

\section{Analytic Procedure}

We used mixed models to analyze main effects of age group and experimental group and their interactions with respect to practice gains and the various transfer effects. These models allowed for the relaxation of the variance homogeneity constraints across age groups. Analyses were conducted with SAS PROC MIXED, and degrees of freedom were calculated with the Kenward-Roger method (Kenward \& Roger, 1997). The alpha level was set to .05 for all analyses.

In this section, the results are presented in four parts. First, we report findings regarding practice gains in the targeted spatial two-back task. Second, we describe the results regarding near transfer effects to both more demanding same modality and different modality $n$-back tasks. Third, we report results regarding far transfer to complex span tasks. Fourth, we summarize results regarding the maintenance of practice gains and transfer effects. 


\section{Practice Gains in the Spatial Two-Back Task}

Both age groups improved their spatial working memory performance substantially in the regular condition and the mental shifting condition of the spatial two-back task over 45 daily practice sessions (see Figure 2). Practice gain was computed as the difference between posttest and baseline performance and was analyzed separately for accuracy and reaction times (RTs). For accuracy, results of the overall analysis of practice gain with age group, task condition, and treatment group as factors showed significant main effects of age group, $F(1,78)=12.62, p<.05$, and task condition, $F(1,75)=15.66, p<.05$. Practice gains were larger in the more demanding condition that required mental shifting. Practice gains were substantially larger than test-retest effects, as indicated by the significant treatment group effect for practice gains, $F(1,78)=36.88, p<.05$. All two-way interactions and the three-way interaction were significant. The main effect of age group and the significant three-way interactions of age group, task condition, and treatment group indicate greater gains in older adults than in younger adults. However, given a potential functional ceiling effect in younger adults after practice, this interaction should be interpreted cautiously.

For RT, results of the overall analysis with the same factors showed significant main effects of treatment group, $F(1,80)=$ $82.20, p<.05$, and task condition, $F(1,68)=13.88, p<.05$. The two-way interactions were all significant: $F(1,80)=13.29, p<$ .5 , for the Age $\times$ Treatment interaction; $F(1,69)=12.55, p<.05$, for the Age $\times$ Task Condition interaction; and $F(1,69)=14.53$, $p<.05$, for the Treatment $\times$ Task Condition interaction. The three-way interaction was not significant, $F(1,68)=1.97$. Practice improved processing speed much beyond the test-retest effect, and performance enhancement was larger in the more demanding condition. There was no reliable main effect of age group, $F(1,80)=$ 3.1 , and this indicated that younger adults and older adults did not differ reliably in processing speed improvement.

\section{Near Transfer to Same Modality but More Demanding Spatial Three-Back Task}

To analyze the effect of practicing the spatial two-back task on performance in the more demanding spatial three-back task, we compared the differences between baseline and posttest performances in the treatment and control groups. For accuracy, results of an analysis with age group and treatment group as factors showed a significant main effect of treatment group, $F(1,83)=$ $27.58, p<.05$. All other effects were not significant. For RT, we found a significant main effect of treatment group, $F(1,77)=$ $51.39, p<.05$ (see Figure 3). Again, all other effects were not significant. We also compared the effects of practicing the spatial two-back task on the spatial three-back task and the spatial zeroback task by contrasting these two tasks directly and found significant interactions between treatment group and the task contrast for RT, $F(1,76)=37.84, p<.05$, as well as for accuracy, $F(1$,

\section{Regular Condition}
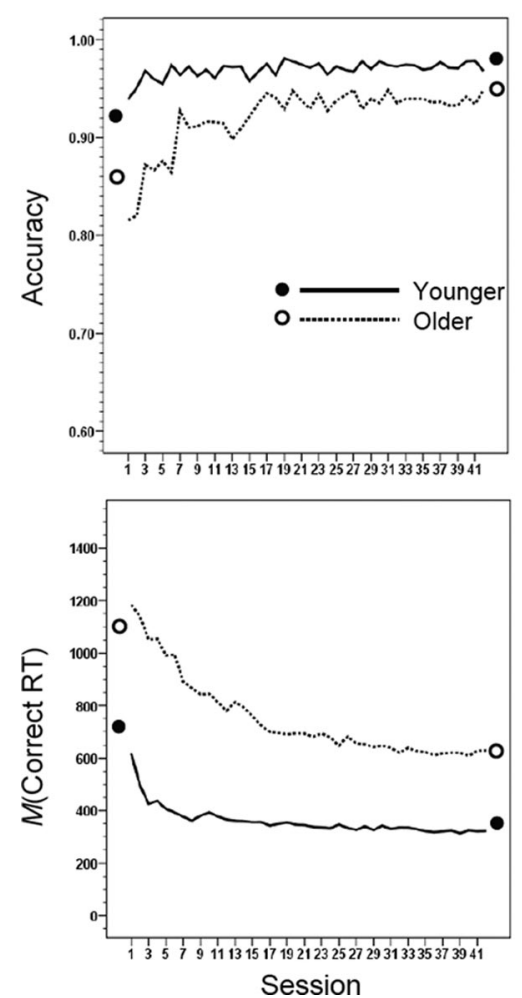

\section{Shifting Condition}
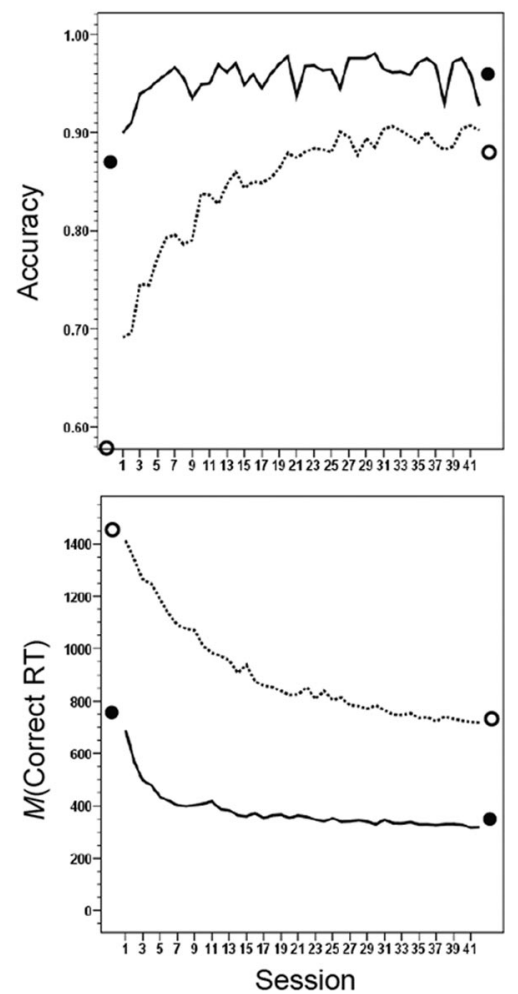

Figure 2. Mean practice curves of the spatial two-back task in two conditions for younger and older adults. Circles denote mean performance levels at pre- and posttest. RT $=$ reaction time (in $\mathrm{ms}$ ). 


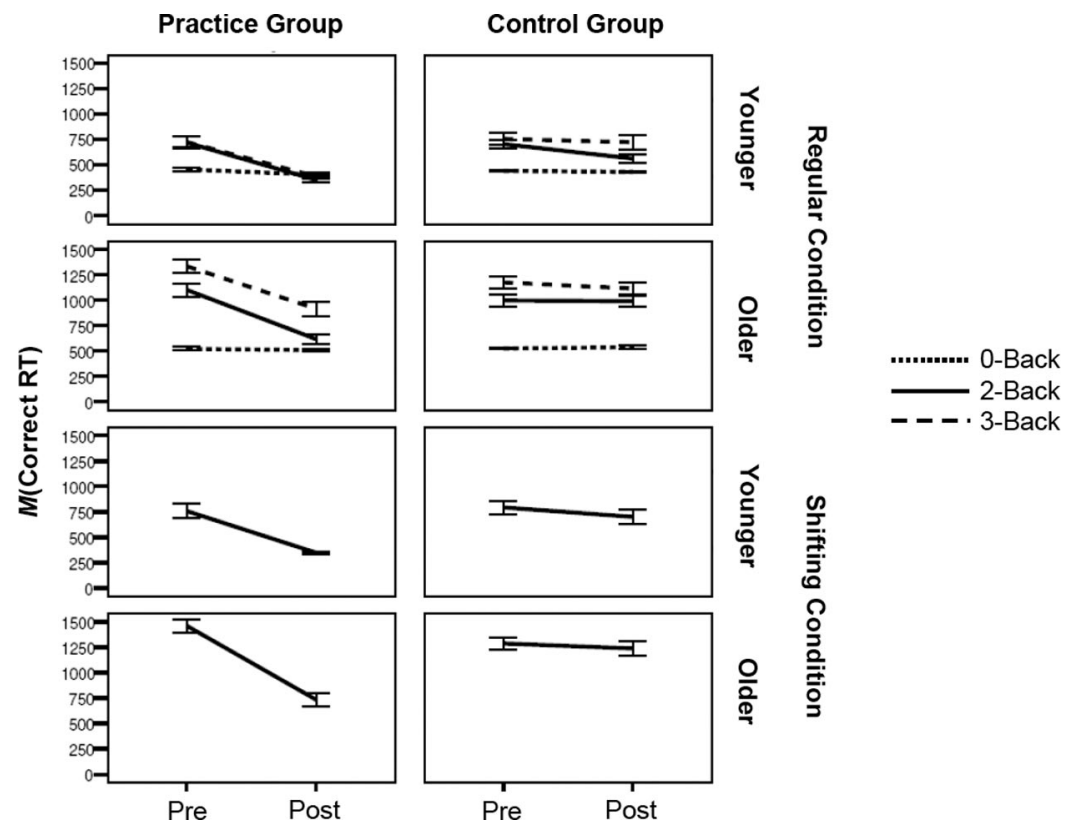

Figure 3. Mean reaction times (in milliseconds) at pretest and posttest for four tasks: spatial two-back, spatial three-back, spatial zero-back, and spatial two-back with mental shifting. The spatial two-back and spatial two-back with shifting tasks were practiced for 45 days in the practice group. The spatial three-back and spatial zero-back assessed near transfer and non-memory-specific transfer. Error bars show standard errors of the means. $\mathrm{RT}=$ reaction time.

$81.9)=28.97, p<.05$. These results indicate that transfer was larger for the three-back spatial working memory task than for the spatial zero-back task (i.e., the simple decision speed task). Therefore, transfer to the three-back spatial working memory task could not be accounted for by improvements in simple sensorimotor aspects of decision speed that are common in both tasks (see summary of results in Figure 3). The corresponding three-way interaction was not significant, and this fact indicates that this observation holds for both age groups. In both age groups, the effect size of the transfer effects and practice gains qualify as moderate to large by conventional standards (for effect sizes, see later summary highlights in Figures 5 and 6 for RTs and accuracy, respectively).

\section{Near Transfer to Different Modality Numerical Two-Back and Three-Back Tasks}

In addition, we analyzed transfer effects to three numerical $n$-back tasks: a regular two-back task without additional processing, a two-back task with the additional processing of adding one to the presented number, and a regular three-back task. In terms of RTs, significant effects of treatment group were found in the regular two-back task, $F(1,79.9)=14.34, p<.05$; in the twoback plus processing task, $F(1,81.3)=22.72, p<.05$; and the three-back task, $F(1,70.6)=13.92, p<.05$. All other effects were not significant (see summary of results in Figure 4). Thus, practice of a spatial working memory task transferred to tasks sharing similar requirements of processing operations but with different stimulus modality.
With regard to accuracy, no significant effect was found in the regular two-back task. In the two-back with processing task, we found reliable effects for age group, $F(1,77.6)=18.39, p<.05$, and treatment group, $F(1,77.6)=49.44, p<.05$; there was a reliable age Group $\times$ Treatment Group interaction, $F(1,77.6)=$ $26.76, p<.05$. The significant age group main effect indicates that the transfer effect to the two-back plus processing task was larger in older adults. With respect to the three-back task, only the treatment group effect was significant, $F(1,83)=27.58, p<.05$ (see Figures 5 and 6 for a summary of effect sizes).

To further evaluate the specificity of the observed effects, we contrasted transfer effects in all three tasks to the simple numerical decision speed (i.e., the numerical zero-back) task. In terms of RT, a significant interaction of Treatment $\times$ Task was found for the numerical two-back task, $F(1,75.6)=37.84, p<.05$; the numerical two-back plus processing task, $F(1,79.2)=9.92, p<.05$; and the numerical three-back task, $F(1,71.6)=6.07, p<.05$. As for accuracy, this interaction was significant for the regular two-back task, $F(1,83)=28.37, p<.05$, and was marginally significant for two-back plus processing, $F(1,62.8)=3.69, p=.058$, and for the three-back task, $F(1,63.6)=3.69, p=.059$. These results indicate that transfer effects were larger for $n$-back tasks than for the simple decision speed task (see Figure 4). None of the threeway interactions were statistically reliable.

\section{Lack of Far Transfer to Complex Span Tasks}

Performance improvements in operation and rotation span were comparable for the experimental and control groups. For neither 


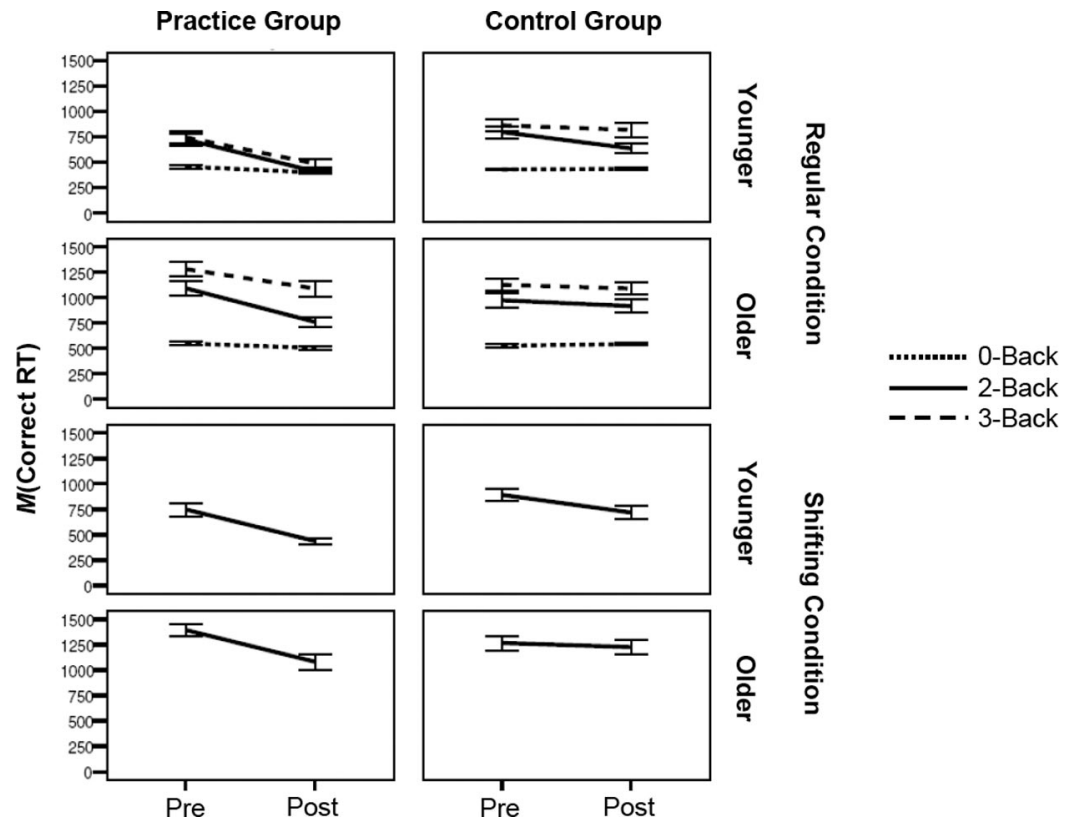

Figure 4. Mean reaction times (in milliseconds) at pretest and posttest for the numerical two-back, numerical three-back, numerical zero-back, and numerical two-back with mental shifting tasks. All four tasks assessed near transfer to tasks with different memory content. Error bars show standard errors of the means. RT = reaction time.

task was the effect of treatment group significant: $F(1,75.8)=$ 0.64 for operation span and $F(1,75.8)=0.23$ for rotation span. For rotation span, the three-way interaction of age group, experimental group, and occasion, $F(1,75.8)=9.33, p<.05$, reflected a significant decrease of performance from pre- to posttest for older participants in the experimental group; there was a significant interaction of occasion and experimental group within the older age group, $F(1,39)=9.87 ; p<.05$. We have no explanation for this decrease in performance. Overall, these results provide no evidence for positive transfer to complex span tasks.

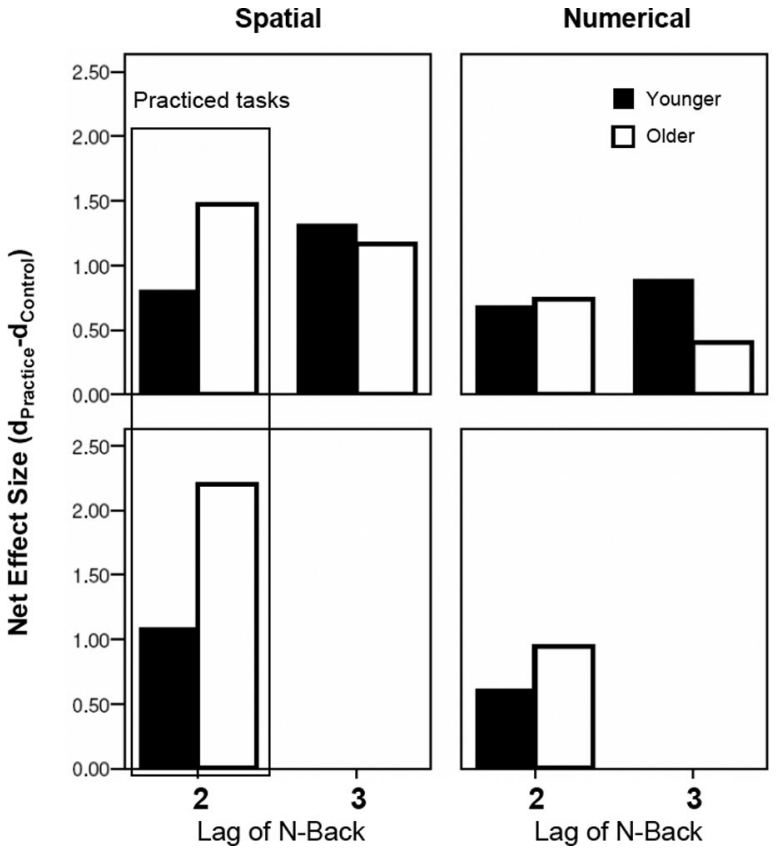

Figure 5. Summary of net effect sizes (Cohen's $d$ for practice group minus control group) of reaction times for practice gains and transfer effects in near transfer tasks. Effect sizes reflect gains in performance speed.

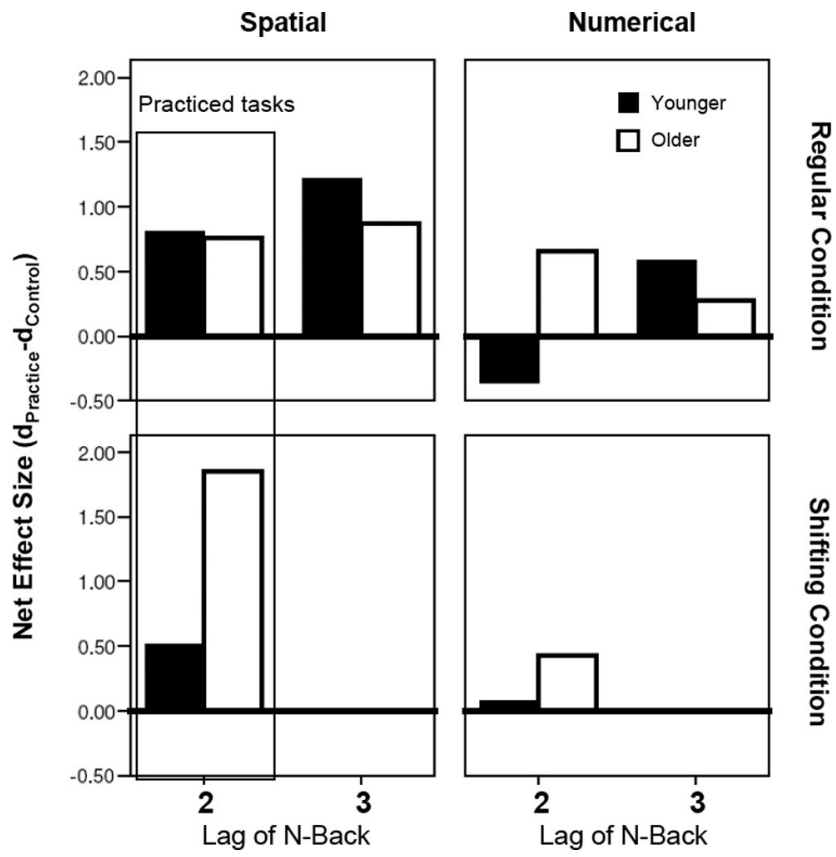

Figure 6. Summary of net effect sizes (Cohen's $d$ for practice group minus control group) of accuracy for practice gains and transfer effects in near transfer tasks. Effect sizes reflect gains in accuracy. 


\section{Maintenance of Practice and Transfer Gains}

The maintenance session, on average, took place 3 months after the posttest session. Maintenance of performance was assessed with respect to posttest performance. There was a reliable main effect of age group for the practiced task (i.e., the two-back spatial task), $F(1$, $21)=8.38, p<.05$, and this indicated that overall performance accuracy was better maintained in younger adults than in older adults (see solid lines in Figure 7). Effects of task condition and its interaction with age group were not significant. A similar pattern was observed for RTs: RTs of the practiced task were better maintained in younger adults than in older adults, $F(1,18)=44.49, p<.05$. Older adults' performance drop in response speed was particularly pronounced in the more demanding mental-shift condition, as revealed by a significant Age Group $\times$ Task Condition interaction, $F(1,14)=$ $4.54, p<.05$ (see solid lines in Figure 8). However, it is important to note that, although older adults did not maintain their performance as well as did younger adults, their performance at maintenance was still considerably higher than their performance at pretest (see the comparison to performance at pretest in Figures 7 and 8).

We also examined whether transfer effects to the more demanding three-back spatial task and the numerical $n$-back tasks with a different stimulus modality were maintained. As shown in Figures 7 and 8, transfer effects were maintained over 3 months in both age groups, in terms of both accuracy and speed.

\section{Discussion}

\section{Summary of Findings}

Working memory is central to many cognitive functions and declines substantially from early to late adulthood. Hence, empirical evidence on working memory plasticity in younger and older adults has important conceptual and practical implications. Here, we explored adult age differences in three indicators of working memory plasticity: practice gains, transfer, and maintenance. We found that 45 days of 15-min practice in two spatial two-back tasks considerably improved performance on the practiced tasks in both younger and older adults. It should be noted that maximum performance of older adults after extensive practice was still lower than was that of younger adults and approached the level of younger adults at baseline (see Figure 2).

Furthermore, the 45-day intervention resulted in positive transfer to spatial three-back performance as well as to numerical two-back and three-back performance. Contrary to our expectations, the observed transfer effects were not larger in younger adults than in older adults Control analyses with spatial and numerical simple decision speed tasks revealed that the observed transfer effects went beyond improvements in the psychomotor speed component of $n$-back performance. At the same time, we found no evidence in either age group for far transfer to complex span tasks.

In both age groups, practice gains were maintained over 3 months, with younger adults showing greater maintenance than did older adults. Despite the drop in performance from posttest to maintenance assessment, older adults still performed the practiced $n$-back tasks considerably more accurately and and at about twice the speed than they had at pretest. Moreover, participants in both age groups were able to maintain improvements on the near transfer tasks: the spatial threeback, the numerical two-back, and the numerical three-back tasks.

\section{Improvements in Working Memory Performance: Skill Alone or Capacity Also?}

A pivotal question in cognitive intervention research concerns the scope of experience-dependent alterations in brain and behav-
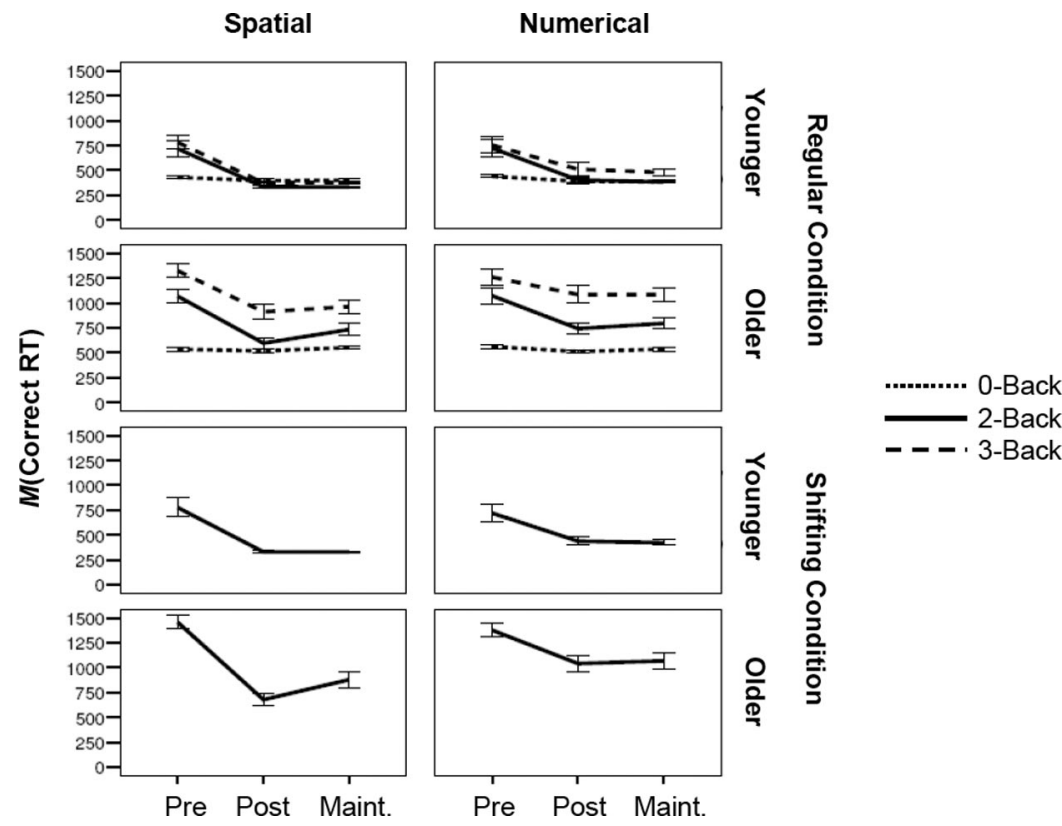

Figure 7. Mean performance accuracy of the experimental groups at pretest, posttest, and after 3 months (maintenance) for spatial and numerical $n$-back performance at two levels of processing demands. Error bars show standard errors of the means. $\mathrm{RT}=$ reaction time (in milliseconds). 

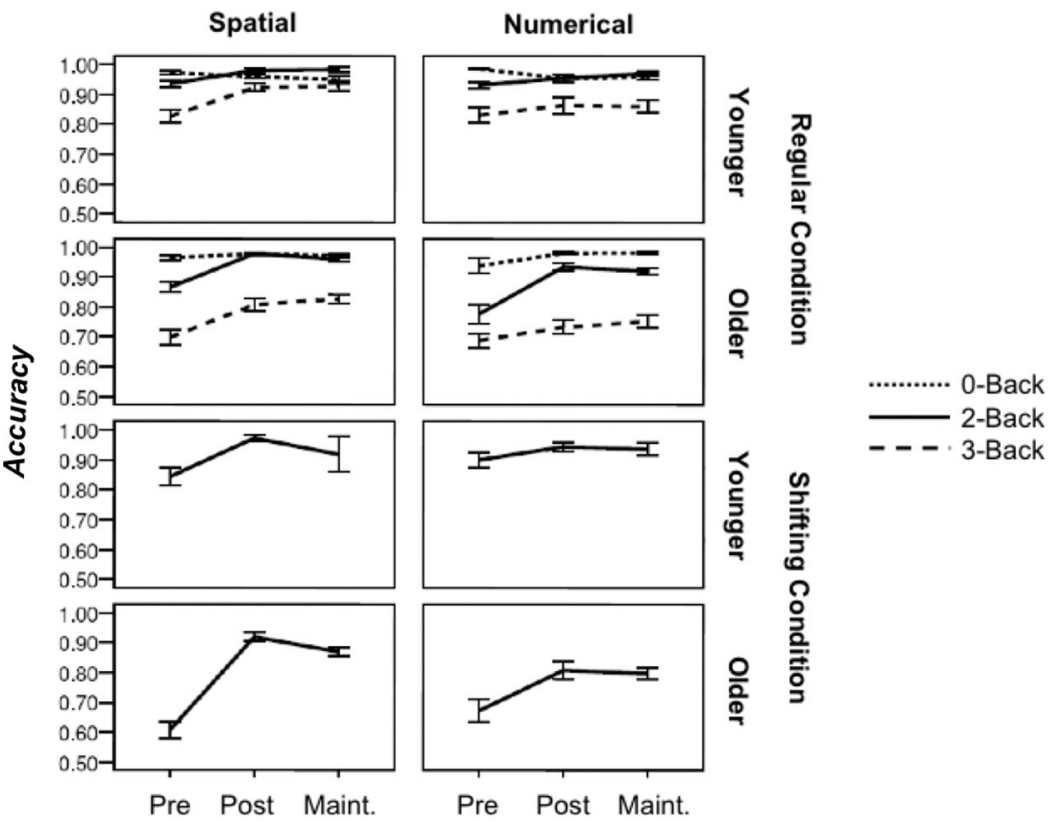

Figure 8. Mean reaction times in milliseconds of the experimental groups at pretest, posttest, and after 3 months (maintenance) for spatial and numerical $n$-back performance at two levels of processing demands. Error bars show standard errors of the means.

ior. Does the intervention primarily foster the acquisition of elements of skill (e.g., Thorndike, 1906), or does it generate cognitive resources and improve cognitive abilities? The pattern of transfer and maintenance observed in this study does not permit an unequivocal answer to this question. We observed substantial transfer to task conditions with higher capacity demands and different stimulus modality. It is tempting to portray these improvements as practice-induced alterations in working memory capacity in general. However, it needs to be noted (a) that the tasks showing positive transfer shared task requirements with the practiced task and (b) that $n$-back practice did not reliably transfer to the complex span tasks.

We can think of at least three explanations for the absence of reliable far transfer in the present study. First, practice-induced changes in working memory capacity, if they occurred in the first place, may not have been large enough to result in far transfer. Second, the sensitivity of the complex span tasks may be too low to capture practice-induced capacity changes. Third, correlations between the $n$-back task and more complex span measures are variable, with some studies reporting relatively low correlations (e.g., Jaeggi et al., 2008; Kane, Conway, Miura, \& Colflesh, 2007) and others reporting high correlations (e.g., Schmiedek, Hildebrandt, Lövdén, Wilhelm, \& Lindenberger, 2008). Therefore, it is difficult to judge whether the complex working memory tasks showed no signs of transfer because $n$-back practice did not affect working memory ability or because working memory is subdivided into different components, so that transfer would have been observed for tasks that are procedurally different from $n$-back tasks but that involve more similar components of working memory (e.g., updating; see Dahlin et al., 2008). Further studies with a more fine-grained continuum of transfer tasks within the larger do- main of working memory are needed to provide more conclusive answers to the skill versus capacity question. In future, researchers should also explore more variable and extensive maintenance regimes, with multiple follow-ups and continued practice, to examine whether the aging-related losses in the ability to maintain practice gains and near-transfer effects observed in the present study can be overcome.

\section{Comparison of Transfer Findings With Results Obtained by Dahlin et al. (2008)}

In this study, near-transfer effects did not differ reliably between younger adults and older adults. In contrast, Dahlin et al. (2008) reported negative age differences in transfer to a three-back memory task after extensive practice in a working-memory updating task. In our view, there are several potential reasons for this difference in findings. First, our intervention program lasted for 45 days with 15-min practice each day, whereas the program used in the Dahlin et al. (2008) study involved three 45-min sessions per week for 5 weeks. The large number of distributed daily practices in the present study may have facilitated the consolidation of plastic changes in older adults (e.g., Spreng, Rossier, \& Schenk, 2002). Second, the overlap in elements of skill between the practiced and the near-transfer tasks may have been greater in the present study than in the Dahlin et al. study. Also, the practice task in Dahlin et al. was a letter memory task that differed from our three-back transfer task in terms of stimulus modality, presentation rate, and response format. Given that near transfer in the present study required improvements in tasks with higher capacity demands or different stimulus modality, we hold that the second reason is less likely. 


\section{Age-Related Decrement in Maintenance and Reactivation} of Skilled Performance

The one clear negative age difference in our findings is older adults' decrement in the maintenance of practice gains. Although older adults maintained their performance at a relatively high level in comparison with their initial baseline performance 3 months after the practice intervention had terminated, their performance at the maintenance session in comparison to posttest dropped more than did that of younger adults (cf. MacDonald, Stigsdotter Neely, Derwinger, \& Bäckman, 2006). In other words, practice-induced memory skill may be more stable and robust in younger than in older adults. This finding indicates that interspersed short, refresh practice sessions after the termination of intensive intervention programs may be necessary to maintain long-term intervention effects in skilled working memory performance for older adults. In the area of episodic memory training also, older adults showed a lower level of maintenance performance; however, their performance could be reactivated after refamiliarization with the learned mnemonics (Brehmer et al., 2008).

\section{Limitations of the Present Study}

Our findings need to be considered in light of a few caveats. First, the difficulty level of the practiced task was not adaptively adjusted for each individual (cf. Brehmer et al., 2007) across the 45 daily practice sessions. Whereas the task remained challenging for older adults across the entire 45 days of intervention, after 1 week of practice younger adults had reached the functional ceiling in performance accuracy. Given this limitation, adult age differences in practice gains and transfer effects may have been underestimated in our findings, as the potential range for improvement was more restricted for younger adults. It should be noted that although we did not find negative adult age differences in practice gains or transfer, the maximum levels of performance reached by older adults were much lower than those obtained younger adults. In fact, after 45 daily-practice sessions, at posttest older adults performed at about the same level as did younger adults at pretest. Second, although the use of no-contact control groups is not uncommon in most cognitive intervention studies, we could not preclude possible beneficial effects of daily social interactions on working memory improvements. Third, given the use of a nocontact control group, it made little sense for us to assess skill maintenance in the control group, because participants in this group did not receive any intervention. The maintenance of transfer effects we observed could, thus, not be compared directly with those of a control group. However, in the current design, comparison of the experimental group's performances on the transfer tasks at the maintenance session with those of the control group at the posttest session clearly demonstrates that the observed transfer effects were beyond test-retest effects. In particular, for both younger and older adults, the experimental group performed better on all transfer tasks at the 3-month follow-up than did the control group at posttest.

\section{Outlook: Combining Behavioral and Neural Levels of Analysis}

Research on neural correlates of working memory practice and transfer in humans is developing at a rapid rate. The dorsolateral prefrontal cortex and the striatum interact closely in a corticostriatal network (Pasupathy \& Miller, 2005; Postuma \& Dagher, 2006; Seamans \& Yang, 2004), and this network plays a central role in executive control and working memory (Miller \& Cohen, 2001). The striatum is involved in selecting relevant information into working memory (i.e., gating; cf. McNab \& Klingberg, 2008), and it appears to mediate improvement in the updating function of working memory (Dahlin et al., 2008). Therefore, age-related changes in the striatum and in corticostriatal connections may provide further insights into adult age differences in the different aspects of working memory plasticity. Striatal volume decreases with age (Raz et al., 2005), and dopaminergic modulation undergoes substantial decline (for review, see Bäckman, Nyberg, Lindenberger, Li, \& Farde, 2006). Neurocomputational models have been proposed to capture the neuromodulatory dynamics of the corticostriatal network (Durstewitz \& Seamans, 2002; see also O'Reilly, 2006, for review), and negative adult age differences in episodic memory plasticity have been modeled by attenuating the stochastic gain tuning of neural networks to simulate aging-related deficit in dopaminergic modulation (Li, Lindenberger, \& Sikström, 2001; Li, Brehmer, Shing, Werkle-Bergner, \& Lindenberger, 2006). The combination and extension of these modeling approaches offers a promising route toward a more thorough understanding and theory-guided predictions of adult age changes in working memory plasticity.

\section{References}

Bäckman, L., Nyberg, L., Lindenberger, U., Li, S.-C., \& Farde, L. (2006). The correlative triad among aging, dopamine, and cognition: Current status and future prospects. Neuroscience \& Biobehavioral Reviews, 30, 791-807.

Baddeley, A. (2007). Working memory, thought, and action. New York: Oxford University Press.

Ball, K., Berch, D. B., Helmers, K. F., Jobe, J. B., Leveck, M. D., et al. (2002). Effects of cognitive training interventions with older adults. Journal of the American Medical Association, 288, 2271-2281.

Baltes, P. B., \& Kliegl, R. (1992). Further testing of limits of cognitive plasticity: Negative age differences in a mnemonic skill are robust. Developmental Psychology, 28, 121-125.

Baltes, P. B., \& Lindenberger, U. (1988). On the range of cognitive plasticity in old age as a function of experience: 15 years of intervention research. Behavior Therapy, 19, 283-300.

Baltes, P. B., Lindenberger, U., \& Staudinger, U. M. (2006). Lifespan theory in developmental psychology. In W. Damon \& R. M. Lerner (Eds.), Handbook of child psychology: Vol. 1. Theoretical models of human development (6th ed., pp. 569-664). New York: Wiley.

Barrett, L. F. S., Tugade, M. M., \& Engle, R. W. (2004). Individual differences in working memory capacity and dual-process theories of the mind. Psychological Bulletin, 130, 553-573.

Bialystok, E., \& Craik, F. I. M. (2006). Lifespan cognition: Mechanisms of change. Oxford, England: Oxford University Press.

Borella, E., Carretti, B., \& de Beni, R. (2008). Working memory and inhibition across the adult lifespan. Acta Neuropsychologica, 128, 33-44.

Brehmer, Y., Li, S.-C., Müller, V., von Oertzen, T., \& Lindenberger, U. (2007). Memory plasticity across the life span: Uncovering children's latent potential. Developmental Psychology, 43, 465-478.

Brehmer, Y., Li, S.-C., Straube, B., Stoll, G., von Oertzen, T., Müller, V., \& Lindenberger, U. (2008). Comparing memory skill maintenance across the life span: Preservation in adults, increase in children. Psychology and Aging, 23, 227-238. 
Brehmer, Y., Westerberg, H., Söderman, D., Fischer, H., \& Bäckman, L. (2008, April). Neural correlates of working memory training in younger and older adults: An fMRI study. Poster session presented at the Cognitive Aging Conference, Atlanta, GA.

Craik, F. I. M. (1983). On the transfer of information from temporary to permanent memory. Philosophical Transactions of the Royal Society of London, B, 302, 341-359.

Dahlin, E., Stigsdotter-Neely, A., Larsson, A., Bäckman, L., \& Nyberg, L. (2008). Transfer of learning after updating training mediated by the striatum. Science, 320, 1510-1512.

Durstewitz, D., \& Seamans, J. K. (2002). The computational role of dopamine D1 receptors in working memory. Neural Networks, 15, 561-572.

Hertzog, C., Kramer, A. F., Wilson, R. S., \& Lindenberger, U. (in press). Enrichment effects on adult cognitive development: Can the functional capacity of older adults be preserved and enhanced? Psychology in the Public Interest.

Huxhold, O. (2007). Processing fluctuations in postural control: Relations to adult age and fluctuations in cognition. (Doctoral dissertation, Free University Berlin, 2007). Retrieved from http://www.diss.fu-berlin.de/ diss/receive/FUDISS_thesis_000000002856

Jaeggi, S. M., Buschkuehl, M., Jonides, J., \& Perrig, W. J. (2008). Improving fluid intelligence with training on working memory. Proceedings of the National Academy of Science, 105, 6829-6833.

Kane, M. J., Conway, A. R. A., Miura, T. K., \& Colflesh, G. J. (2007). Working memory, attention control, and the $N$-back task: A question of construct validity. Journal of Experimental Psychology: Learning, Memory, and Cognition, 33, 615-622.

Kane, M. J., Hambrick, D. Z., Tuholski, S. W., Wilhelm, O., Payne, T. W., \& Engle, R. E. (2004). The generality of working-memory capacity: A latent-variable approach to verbal and visuospatial memory span and reasoning. Journal of Experimental Psychology: General, 133, 189217

Karbach, J., \& Kray, J. (2008). How useful is executive control training? Age differences in near and far transfer of task-switching training. Manuscript submitted for publication.

Kenward, M. G., \& Roger, J. H. (1997). Small sample inference for fixed effects from restricted maximum likelihood. Biometrics, 53, 983-997.

Kliegl, R., Smith, J., \& Baltes, P. B. (1989). Testing-the-limits and the study of adult age differences in cognitive plasticity of a mnemonic skill. Developmental Psychology, 25, 247-256.

Klingberg, T., Fernell, E., Olesen, P.J., Johnson, M., Gustafsson, P., Dahlstrom, K., et al. (2005). Computerized training of working memory in children with ADHD: A randomized, controlled trial. Journal of the American Academy of Child and Adolescent Psychiatry, 44, 177-186.

Kramer, A. F., \& Willis, S. L. (2002). Enhancing the cognitive vitality of older adults. Current Directions in Psychological Science, 11, 173-176.

Kyllonen, P. C., \& Christal, R. E. (1990). Reasoning ability is (little more than) working-memory capacity?! Intelligence, 14, 389-433.

Lerner, R. M. (1984). On the nature of human plasticity. Cambridge, England: Cambridge University Press.

Li, S.-C. (2003). Biocultural orchestration of developmental plasticity across levels: The interplay of biology and culture in shaping the mind and behavior across the life span. Psychological Bulletin, 129, 171-194.

Li, S.-C., Brehmer, Y., Shing, Y. L., Werkle-Bergner, M., \& Lindenberger, U. (2006). Neuromodulation of associative and organizational plasticity across the life span: Empirical evidence and neurocomputational modeling. Neuroscience \& Behavioral Reviews, 30, 775-790

Li, S.-C., Lindenberger, U., \& Sikström, S. (2001). Aging cognition: From neuromodulation to representation. Trends in Cognitive Sciences, 5, $479-486$.

Lindenberger, U., \& Baltes, P. B. (1995). Testing-the-limits and experimental simulation: Two methods to explicate the role of learning in development. Journal of Human Development, 38, 349-360.
Lindenberger, U., Li, S.-C., \& Bäckman, L. (2006). Methodological and conceptual advances in the study of brain-behavior dynamics: A multivariate lifespan perspective. Neuroscience \& Biobehavioral Reviews, 30, 713-892.

MacDonald, S. W. S., Stigsdotter Neely, A., Derwinger, A., \& Bäckman, L. (2006). Rate of acquisition, adult age, and basic cognitive abilities predict forgetting: New views on a classic problem. Journal of Experimental Psychology: General, 135, 368-390.

McNab, F., \& Klingberg, T. (2007). Prefrontal cortex and basal ganglia control access to working memory. Nature Neuroscience, 11, 103-107.

Miller, E. K., \& Cohen, J. D. (2001). An integrative theory of prefrontal cortex function. Annual Review of Neuroscience, 24, 167-202.

O'Reilly, R., \& Frank, M. (2006). Making working memory work: A computational model of learning in the frontal cortex and basal ganglia. Journal of Cognitive Neuroscience, 18, 22-32.

O'Reilly, R. C. (2006). Biologically based computational models of highlevel cognition. Science, 314, 91-94.

Oberauer, K. (2005). Binding and inhibition in working memory: Individual and age differences in short-term recognition. Journal of Experimental Psychology: General, 134, 368-387.

Olesen, P., Westerberg, H., \& Klingberg, T. (2004). Increased prefrontal and parietal brain activity after training of working memory. Nature Neuroscience, 7, 75-79.

Pasupathy, A., \& Miller, E. K. (2005). Different time courses of learningrelated activity in the prefrontal cortex and striatum. Nature, 433, 873876.

Paxton, J. L., Barch, D. M., \& Racine, C. A. (2008). Cognitive control, goal maintenance, and prefrontal function in healthy aging. Cerebral Cortex, 18, 1010-1028.

Postuma, R. B., \& Dagher, A. (2006). Basal ganglia functional connectivity based on a meta-analysis of 126 positron emission tomography and functional magnetic resonance imaging publications. Cerebral Cortex, $16,1508-1521$.

Raz, N., Lindenberger, U., Rodrigue, K. M., Kennedy, K. M., Head, D., et al. (2005). Regional brain changes in aging healthy adults: General trends, individual differences, and modifiers. Cerebral Cortex, 18 $1676-1689$.

Rebok, G. W., Carlson, M. C., \& Langbaum, J. B. S. (2007). Training and maintaining memory abilities in healthy older adults: Traditional and novel approaches. Journal of Gerontology, 62B, 53-61.

Röcke, C. (2006). Intraindividual variability in positive and negative affect: Age-related and individual differences in magnitude and coupling with cognitive performance. (Doctoral dissertation, Free University Berlin, 2006). Retrieved from http://www.diss.fu-berlin.de/diss/receive/ FUDISS_thesis_000000002464

Salthouse, T. A., \& Pink, J. E. (2008). Why is working memory related to fluid intelligence. Psychonomic Bulletin \& Review, 15, 364-371.

Schmiedek, F., Hildebrandt, A., Lövdén, M., Wilhelm, O., \& Lindenberger, U. (2008). Complex span versus binding tasks of working memory: The gap is not that deep. Manuscript submitted for publication.

Seamans, J. K., \& Yang, C. R. (2004). The principle features and mechanisms of dopamine modulation in the prefrontal cortex. Progress in Neurobiology, 74, 1-57.

Shah, P., \& Miyake, A. (1996). The separability of working memory resources for spatial thinking and language processing: An individual differences approach. Journal of Experimental Psychology: General, $125,4-27$.

Shing, Y. L., Werkle-Bergner, M., Li, S.-C., \& Lindenberger, U. (2008). Associative and strategic components of episodic memory: A life span dissociation. Journal of Experimental Psychology: General, 137, 495513.

Singer, T., Lindenberger, U., \& Baltes, P. B. (2003). Plasticity of memory for new learning in very old age: A story of major loss? Psychology and Aging, 18, 306-317. 
Spreng, M., Rossier, J., \& Schenk, F. (2002). Spaced training facilitates long-term retention of place navigation in adult but not in adolescent rats. Behavioural Brain Research, 128, 103-108.

Stigsdotter Neely, A., \& Bäckman, L. (1993). Long-term maintenance of gains from memory training in older adults: Two $31 / 2$-year follow-up studies. Journal of Gerontology: Psychological Sciences, 48, P233-P237.

Süss, H.-M., Oberauer, K., Wittmann, W. W., Wilhelm, O., \& Schulze, R. (2002). Working memory capacity explains reasoning ability—And a little bit more. Intelligence, 30, 261-288.

Thorndike, E. L. (1906). Formal discipline. In E. L. Thorndike (Ed.), The principles of teaching based on psychology (pp. 235-256). New York: Seiler.
Turner, M. L., \& Engle, R. W. (1989). Is working memory capacity task dependent? Journal of Memory and Language, 28, 127-154.

Verhaeghen, P., Marcoen, A., \& Goossens, L. (1992). Improving memory performance in the aged through mnemonic training: A meta-analytic study. Psychology and Aging, 7, 242-251.

Received June 16, 2008 Revision received September 30, 2008 Accepted October 2, 2008

\section{Correction to Emery, Hale, and Myerson (2008)}

The article "Age Differences in Proactive Interference, Working Memory, and Abstract Reasoning" by Lisa Emery, Sandra Hale, and Joel Myerson (Psychology and Aging, 2008, Vol. 23, No. 3, pp. 634-645) contained an incorrect DOI. The correct DOI is as follows: 10.1037/a0012577.

DOI: $10.1037 / \mathrm{a} 0014244$ 